\title{
Evolución de la ultramicrofábrica de los peloides en el proceso de maduración
}

\author{
Rafael DELGADO ${ }^{(1)}$, Maria Virginia FERNÁNDEZ-GONZÁLEZ ${ }^{(1)}$, \\ Encarnación GÁMIZ ${ }^{(1)}$, Juan Manuel MARTÍN-GARCÍA ${ }^{(1)}$, Rocío MÁRQUEZ ${ }^{(2)}$, \\ Gabriel DELGADO ${ }^{(1)}$ \\ ${ }^{(1)}$ Departamento de Edafología y Química Agrícola. Facultad de Farmacia. Universidad de \\ Granada. 18071 Campus Universitario de Cartuja, Granada (España). \\ ${ }^{(2)}$ Centro de Instrumentación Científica. Facultad de Farmacia. Universidad de Granada. \\ 18071 Campus Universitario de Cartuja, Granada (España). \\ rdelgado@ugr.es
}

Recibido: 21-01-11

Aceptado: 27-03-11

\section{Resumen}

La ultramicrofábrica ó fábrica estudiada por debajo del límite de resolución del microscopio óptico mediante técnicas de microscopía electrónica de barrido, microanálisis de rayos X y análisis de imagen, ofrece perspectivas nuevas de estudio de los procesos de maduración de los peloides. La ultramicrofábrica ha sido empleada con éxito en Edafología para la resolución de los problemas genéticos. En peloides los estudios sobre la fábrica son escasos, si bien reportan resultados de gran futuro. El objetivo de este trabajo es demostrar, en diversos peloides preparados con aguas minerales y mineromedicinales de la provincia de Granada, la evolución de la ultramicrofábrica con el tiempo de maduración. La fase sólida del peloide fue una mezcla de caolín-bentonita en proporción 9:1 (w:w). Se emplearon aguas de los balnearios y manantiales de La Malahá, Alicún de las Torres, Zújar, Graena y Lanjarón (Capuchina, Salud V y El Salado). Como contraste y muestra blanco se preparó un peloide con agua bidestilada. Los tiempos ensayados fueron uno, tres y seis meses. Las muestras del peloide madurado fueron criofijadas, liofilizadas y metalizadas previo a la observación microscópica. Las fábricas se componen de patrones basados en morfologías laminares de las partículas. Se reconocen fábricas dispersas -tendentes a veces a blocosas ó reticuladas, panal de abejas, castillo de naipes y masivas. La porosidad estimada con análisis de imagen puede superar el 30\%. La fábrica permite establecer que el tiempo óptimo de maduración más frecuente en los peloides estudiados es de tres meses; aunque la fábrica de los peloides a las 48 horas alcanza una destacable evolución.

Palabras claves: Peloide, Fábrica SEM-IA, Tiempo óptimo de maduración, Caolinita, Saponita, Peloides extemporáneos 


\title{
Evolution of the ultramicrofabric of peloids in the maturation process
}

\begin{abstract}
The ultramicrofabric (or fabric studied below the resolution limit of optical microscopy techniques of scanning electron microscopy, X-ray microanalysis and image analysis) offers new perspectives for studying the processes of maturation of peloids. The ultramicrofabric has been used successfully in Soil Science for solving genetic problems. In peloids, studies on the ultramicrofabric are rare, although the reported results have a great future. The aim of this paper is to demonstrate, in various peloids prepared with mineral and mineromedicinal waters of the province of Granada, the evolution of ultramicrofabric with maturation time. The solid phase of the peloid was a mixture of kaolin-bentonite in proportion 9:1 (w: w). We used the spa waters and springs of La Malahá, Alicún de las Torres, Zújar, Graena and Lanjarón (Capuchina, Salud V and El Salado). As a contrast and a blank sample, a peloid with bidistilled water was prepared. The times of maturation were one, three and six months. The matured samples of peloid were cryophilized, lyophilized and metallized prior to microscopic observation. The fabric of peloids is composed of patterns based on laminar morphologies of the particles. Dispersed fabrics are recognized (sometimes prone to form blocks or a lattice), honeycomb, and massive house of cards. The porosity estimated by image analysis can exceed $30 \%$. The fabric study can set that the optimum of maturation time more frequent in the studied peloids is three months; although the fabric of the peloids in 48 hours reach a remarkable evolution.
\end{abstract}

Key words: Peloid, SEM-IA fabric, Optimal maturation time, Kaolinite, Saponite, Extemporaneous peloids

REFERENCIA NORMALIZADA

Delgado R, Fernández-González MV, Gámiz E, Martín-García JM, Márquez R, Delgado G. Evolución de la ultramicrofábrica de los peloides en el proceso de maduración. (Evolution of the ultramicrofabric of peloids in the maturation process). Anal Hidrol Med, 2011, vol. 4, 81-91

\section{INTRODUCCIÓN}

El concepto de fábrica ("fábric", en francés; "gefuge" en alemán) puede encontrarse citado en Kubiena ${ }^{1}$. A inicios de la década de los noventa y como fruto de la experiencia en microscopía electrónica de barrido (SEM), nuestro Grupo de Investigación definió la ultramicrofábrica en suelos como la "Organización total del suelo a escala ultramicroscópica, expresada por la situación espacial de sus constituyentes sólidos y huecos, su forma, frecuencia, tamaño; desde un punto de vista configuracional, funcional, genético, compositivo y de las diversas propiedades y caracteres que pueden influir en la organización". . Consideraba y adoptaba las terminologías de micromorfología de suelos sumarizadas por Bullock et al. ${ }^{3}$, las de ciencias de los materiales e ingenierías ${ }^{4-5}$. Fruto de aquellos fundamentos fueron 
desarrollándose distintas investigaciones para desvelar los procesos genéticos del suelo, destacando el origen de su estructura y su relación a propiedades como el color ó su calidad ${ }^{6}$. Estudios ampliados más tarde con análisis de imagen siendo empleados para desvelar problemas genéticos en cronosecuencias fluviales ${ }^{7}$. En todos estos casos han resultado también cruciales técnicas como el microanálisis de rayos $\mathrm{X}$, al dar información puntual de la composición química, a la par que el conocimiento de la composición mineralógica, propiedades físicas, fisicoquímicas y el apoyo de otras técnicas microscópicas electrónicas y microanalíticas.

La maduración de los peloides es un proceso necesario para que se produzca en la masa del mismo la integración de todos los componentes sólidos con la fase líquida. Periódicamente, la masa reposa y se va removiendo, para finalmente, transformarse en un auténtico peloide gracias a la acción de fenómenos físicos químicos y biológicos. Durante este proceso se debe controlar el equilibrio entre el contenido mineral y orgánico, el estado coloidal, la temperatura, la cantidad de agua que posee o grado de humedad, $\mathrm{pH}$, etc. La finalidad de la maduración es mejorar mediante el paso del tiempo las propiedades del producto, estabilizándolas y en lo posible exaltándolas, además de homogeneizarlas en la masa. Entre los distintos puntos de vista desde los que se ha estudiado la maduración destaca el tipo de fase líquida (aguas sulfurosas, ricas en yodo y bromo, aguas sulfatadas, ricas en calcio o magnesio $)^{8}$ y el tiempo de duración del proceso. Éste último puede variar desde los dos meses ${ }^{9-10}$ hasta dos años ${ }^{8}$. Galzigna ${ }^{9}$ estudió la maduración a $48^{\circ} \mathrm{C}$, en condiciones controladas, observando los cambios físicos (de la matriz mineral) y bioquímicos debidos al crecimiento de colonias de microorganismos termófilos. Por medio de medidas termogravimétricas se identificó un sulfoglicolípido, que puede ser el responsable del efecto antirreumático del peloide y es producido por las especies colonizadoras que se desarrollan durante el periodo de maduración del barro. Se comprobó en este estudio el efecto beneficioso de las aplicaciones de peloides maduros en la osteoporosis posmenopáusica.

Escasos han sido los trabajos que han estudiado el cambio de la ultramicrofábrica de los peloides con el tiempo de maduración y sólo Gámiz et al. ${ }^{11}$ los realizaron en condiciones de preparación de la muestra que garantizasen su similitud a las condiciones reales del barro. Destacar de entre estos resultados que la ultramicrofábrica se modifica con el paso del tiempo, de uno a tres meses. La fase sólida del peloide investigado fue siempre constante. Como fase líquida se empleó agua bidestilada y agua hipersalina de la localidad granadina de La Malahá.

Con estos antecedentes planteamos la presente ponencia cuyo objetivo es investigar, en diversos peloides preparados con aguas mineromedicinales y minerales de la provincia de Granada, la evolución de la ultramicrofábrica con el tiempo de maduración. 


\section{MATERIAL Y MÉTODOS}

\section{Materiales}

Los peloides han sido preparados con 500 gramos de fase sólida, que consiste en una mezcla de caolín y bentonita industriales (Caolín C6A- Bentonita 1) en proporción 9:1 (w:w) y $1000 \mathrm{ml}$ de fase líquida (Tabla 1). La fase sólida se describe en Fernández- González et al ${ }^{12}$ (en este mismo volumen).

La proporción del peloide y el proceso de maduración se ha realizado siguiendo lo descrito en el artículo publicado por Gámiz et $\mathrm{al}^{11}$. Los tiempos de maduración han sido uno, tres y seis meses, obteniendo un total de 24 muestras (Tabla 2), a las que sumar el peloide fabricado con agua bidestilada y estudiado a las 48 horas (muestra B48) donde se supone que la maduración es inicial ó inexistente.

\section{Metodología de estudio de la ultramicrofábrica}

Las muestras de peloide madurado han sido criofijadas, liofilizadas, metalizadas y observadas con microscopio electrónico de barrido. La metodología y equipos empleados están igualmente descritos en detalle por Gámiz et $\mathrm{al}^{11}$.

Un equipo de microanálisis (Espectrómetro de Rayos $\mathrm{X}$ de energía dispersiva (EDR) Röntec 288, M-Serie, Edwin) acoplado al sistema también fue usado. Sobre las imágenes SEM digitalizadas se realizaron medidas del sistema poroso mediante análisis de imagen con el programa "Image J"13.

\section{RESULTADOS}

\section{Caolín y bentonita}

La muestra sólida del caolín (C6A) se observa al SEM (Figura 1) como un conjunto de partículas de morfologías más o menos pseudoesféricas y subredondeadas superficialmente (efecto de la molienda) junto a otras pseudopoliédricas, todas de tamaño limo fino $(2-20 \mu \mathrm{m})$ o incluso limo grueso $(20-50 \mu \mathrm{m})$; compuestas las primeras por agregaciones de partículas de limo fino y arcilla $(<2 \mu \mathrm{m})$, y siendo visible en las segundas (pseudoesféricas) el apilamiento de láminas, cercanas a lo que se conoce clásicamente en la literatura como "stacks" o formas "vermiformes". El microanálisis (espectro incluido en la misma Figura 1), al tratarse mayoritariamente del mineral caolinita, $\mathrm{Si}_{2} \mathrm{O}_{5}(\mathrm{OH})_{4} \mathrm{Al}_{2}$, muestra picos de oxígeno, aluminio y silicio; silicio y aluminio con alturas similares, concordante a la proporción atómica en fórmula: $2 \mathrm{Si}: 2 \mathrm{Al}$. 
Tabla 1 - Descripción general de las aguas mineromedicinales y minerales.

Muestras de peloide estudiadas.

\begin{tabular}{|c|c|c|c|c|}
\hline PROCEDENCIA & $\begin{array}{l}\text { TIPO DE } \\
\text { AGUA }\end{array}$ & $\begin{array}{l}\text { CLASIFICACIÓN } \\
\text { DEL AGUA }\end{array}$ & $\begin{array}{l}\text { TIEMPO DE } \\
\text { MADURACIÓ } \\
\text { N PELOIDE } \\
\text { (MESES) }\end{array}$ & $\begin{array}{l}\text { SIGNATURA } \\
\text { MUESTRA } \\
\text { PELOIDE }\end{array}$ \\
\hline \multirow{3}{*}{$\begin{array}{c}\text { Facultad de } \\
\text { Farmacia - UGR }\end{array}$} & \multirow{3}{*}{$\begin{array}{c}\text { Blanco. } \\
\text { Agua } \\
\text { Bidestilada }\end{array}$} & \multirow{3}{*}{ Oligometálica } & 1 & B1 \\
\hline & & & 3 & B3 \\
\hline & & & 6 & B6 \\
\hline \multirow{3}{*}{$\begin{array}{l}\text { Manantial de La } \\
\text { Malahá, Granada }\end{array}$} & \multirow{3}{*}{$\begin{array}{l}\text { Agua } \\
\text { mineral }\end{array}$} & \multirow{3}{*}{$\begin{array}{l}\text { Hipersalina, sódico- } \\
\text { potásica clorurada }\end{array}$} & 1 & M1 \\
\hline & & & 3 & M3 \\
\hline & & & 6 & M6 \\
\hline \multirow{3}{*}{$\begin{array}{c}\text { Balneario de Alicún } \\
\text { de las Torres, } \\
\text { Granada }\end{array}$} & \multirow{3}{*}{$\begin{array}{l}\text { Agua } \\
\text { minerome- } \\
\text { dicinal }\end{array}$} & \multirow{3}{*}{$\begin{array}{l}\text { Sulfatada cálcica y } \\
\text { magnésica, de mine- } \\
\text { ralización fuerte, } \\
\text { extremadamente dura }\end{array}$} & 1 & A1 \\
\hline & & & 3 & A3 \\
\hline & & & 6 & A6 \\
\hline \multirow{3}{*}{$\begin{array}{c}\text { Baños de Zújar, } \\
\text { Granada }\end{array}$} & \multirow{3}{*}{$\begin{array}{l}\text { Agua } \\
\text { minerome- } \\
\text { dicinal }\end{array}$} & \multirow{3}{*}{$\begin{array}{l}\text { Sulfatada clorurado- } \\
\text { sódica e hipertermal }\end{array}$} & 1 & Z1 \\
\hline & & & 3 & $\mathbf{Z 3}$ \\
\hline & & & 6 & Z6 \\
\hline \multirow{3}{*}{$\begin{array}{l}\text { Balneario de Cortes } \\
\text { y Graena, Granada }\end{array}$} & \multirow{3}{*}{$\begin{array}{l}\text { Agua } \\
\text { minerome- } \\
\text { dicinal }\end{array}$} & \multirow{3}{*}{$\begin{array}{l}\text { Sulfatada cálcica, } \\
\text { hipertermal, de } \\
\text { mineralización fuerte, } \\
\text { extremadamente dura }\end{array}$} & 1 & G1 \\
\hline & & & 3 & G3 \\
\hline & & & 6 & G6 \\
\hline \multirow{3}{*}{$\begin{array}{l}\text { Manantial de Salud } \\
\text { V, Balneario de } \\
\text { Lanjarón, Granada }\end{array}$} & \multirow{3}{*}{$\begin{array}{l}\text { Agua } \\
\text { minerome- } \\
\text { dicinal }\end{array}$} & \multirow{3}{*}{$\begin{array}{l}\text { Bicarbonatada, } \\
\text { clorurada sódica, de } \\
\text { mineralización fuerte }\end{array}$} & 1 & S1 \\
\hline & & & 3 & S3 \\
\hline & & & 6 & S6 \\
\hline \multirow{3}{*}{$\begin{array}{c}\text { Manantial de El } \\
\text { Salado, Balneario de } \\
\text { Lanjarón, Granada }\end{array}$} & \multirow{3}{*}{$\begin{array}{l}\text { Agua } \\
\text { minerome- } \\
\text { dicinal }\end{array}$} & \multirow{3}{*}{$\begin{array}{l}\text { Clorurada, sódica, } \\
\text { cálcica, carbogaseosa } \\
\text { y ferruginosa }\end{array}$} & 1 & E1 \\
\hline & & & 3 & E3 \\
\hline & & & 6 & E6 \\
\hline \multirow{3}{*}{$\begin{array}{l}\text { Manantial de Capu- } \\
\text { china, Balneario de } \\
\text { Lanjarón, Granada }\end{array}$} & \multirow{3}{*}{$\begin{array}{l}\text { Agua } \\
\text { minerome- } \\
\text { dicinal }\end{array}$} & \multirow{3}{*}{$\begin{array}{l}\text { Bicarbonatada, } \\
\text { clorurada, sódica, } \\
\text { cálcica y ferruginosa, } \\
\text { extremadamente dura }\end{array}$} & 1 & C1 \\
\hline & & & 3 & $\mathrm{C3}$ \\
\hline & & & 6 & C6 \\
\hline
\end{tabular}

La imagen SEM del polvo de la Bentonita 1 (Figura 2), muestra fundamentalmente partículas pseudoesféricas y subredondeadas (por molienda), a veces formas planares o más pseudopoliédricas, de unas 20-60 $\mu \mathrm{m}$ de diámetro, que internamente 
parecen ser porosas. Se trataría de agregados de cristales dada la tendencia de estos minerales a presentarse en partículas de pequeña tallas $(10-100 \mathrm{X} \mathrm{nm})^{5}$. El espectro del microanálisis es un reflejo de la fórmula estructural de la especie saponita presente: $\left(\mathrm{Si}_{3,49} \mathrm{Al}_{0,51}\right)\left(\mathrm{Al}_{0,29} \mathrm{Fe}_{0,11} \mathrm{Ti}_{0,03} \mathrm{Mg}_{2,36}\right) \mathrm{O}_{10}(\mathrm{OH})_{2} \mathrm{Mg}_{0,04} \mathrm{Ca}_{0,07} \mathrm{Na}_{0,20} \mathrm{~K}_{0,06}$ [14]. El pico de silicio es el más destacado, como corresponde al contenido atómico, 3,49 átomos por fórmula estructural para media celdilla. Le sigue el magnesio, con 2,40 átomos: $2,36\left(\mathrm{Al}^{\mathrm{VI}}\right)+0,04\left(\mathrm{Mg}^{\mathrm{XII}}\right)$. A continuación aluminio, 0,80 átomos: $0,51\left(\mathrm{Al}^{\mathrm{IV}}\right)+0,29\left(\mathrm{Al}^{\mathrm{VI}}\right)$. Los picos de hierro y sodio también son bien distinguibles: 0,11 y 0,20 átomos, respectivamente. Hemos obtenido pues un encaje relativamente bueno entre los datos de la fórmula estructural y el microanálisis EDX- SEM.

Figura 1 - Imagen SEM del Caolín C6A. Microanálisis EDX

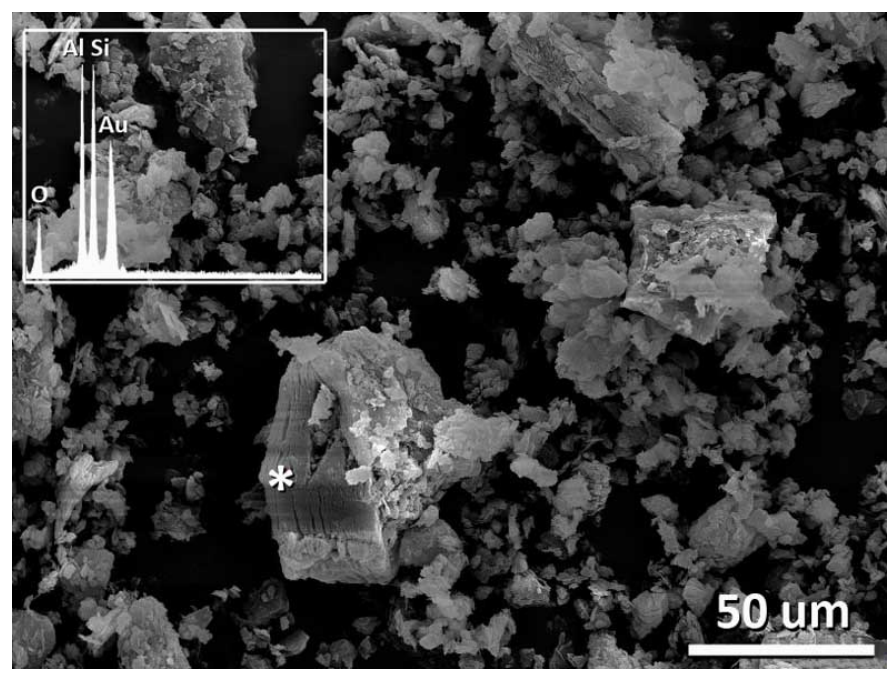

\section{Peloide a las 48 horas}

Contra lo que pudiera pensarse a priori, cuando la mezcla de la fase sólida con el agua bidestilada se produce, el cambio en la morfología SEM es drástico y el peloide constituido presenta una fábrica con cierto grado de organización. Efectivamente, aparecen "clusters" de partículas de unas $5 \mu \mathrm{m}$ de diámetro (Figura 3) formados por agregaciones de partículas mas pequeñas, de talla entre 1-3 $\mu \mathrm{m}$, que se apilan cara-cara, aunque algo desordenadamente (responden algo al concepto de apilamiento "intercrecido"). Los clusters se unen lateralmente dejando huecos entre ellos. Es una fábrica que calificaríamos de "laminar porosa" con cierta anisotropía. La evolución de la fábrica en tan poco espacio de tiempo puede avalar la preparación y utilización de lo que hemos denominado peloides extemporáneos ${ }^{15-16}$, que 
son aquellos fangos que no han sido sometidos a proceso de maduración alguno, y tras su preparación y agitación (para adquirir la consistencia plástica y la homogeneidad deseada), son aplicados en la zona del cuerpo a tratar.

Figura 2 - Imagen SEM de la Bentonita 1. Microanálisis EDX

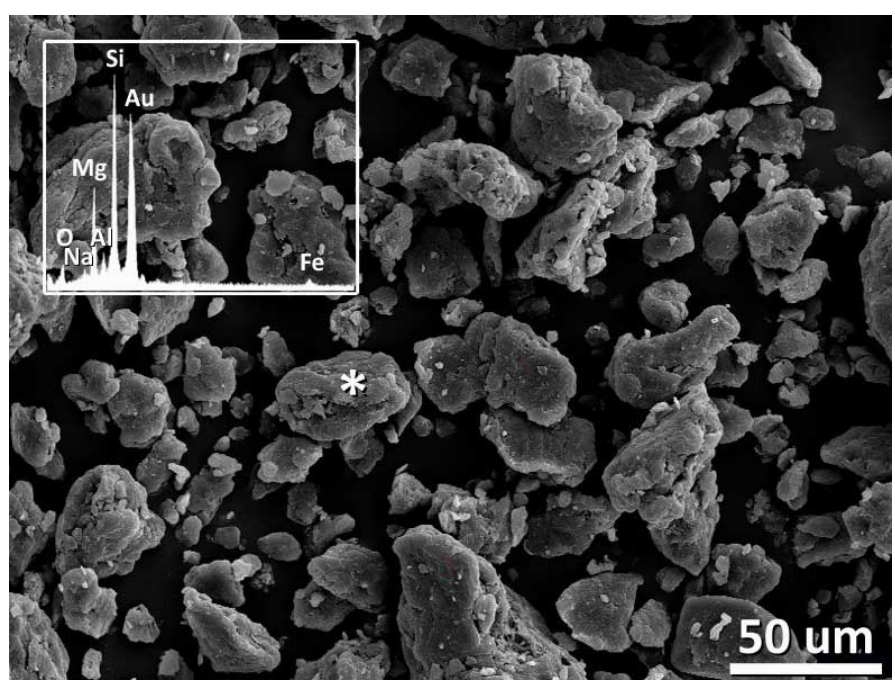

Figura 3 - Imagen SEM de la muestra B48

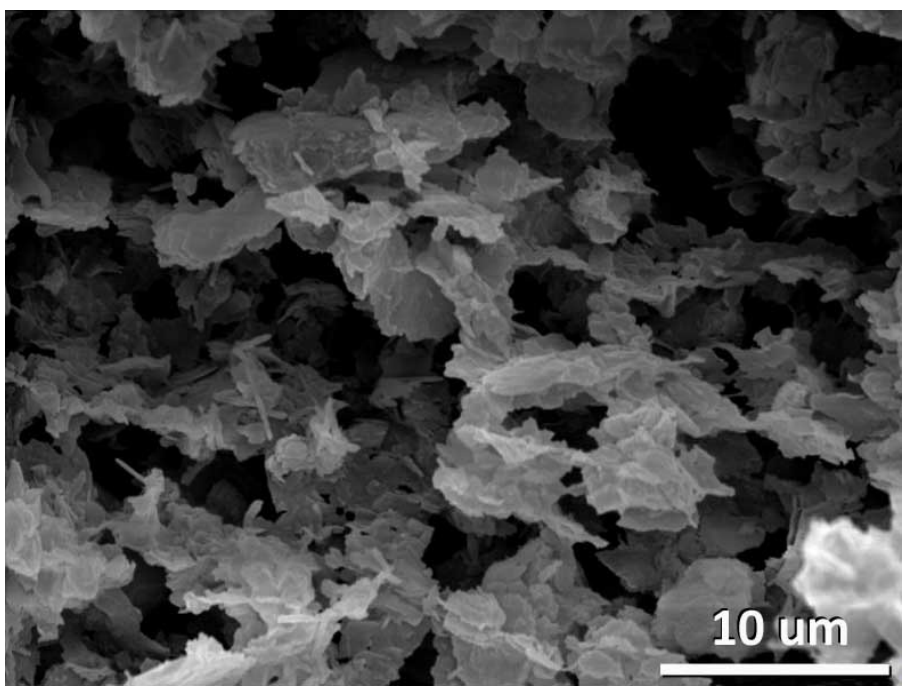




\section{Maduración de los peloides a los tiempos ensayados (uno tres y seis meses)}

Las fábricas encontradas dependen del agua mineromedicinal ó mineral empleada y de los tiempos de maduración. Existe una dependencia pues entre la fábrica con la maduración. Respecto al patrón morfogenético, se han descrito diversos basados todos ellos en la morfología plana de las partículas de los minerales de la fase sólida, mayoritariamente, caolinita. Se describen casos que hemos denominado fábricas "laminares dispersas", con tendencia a veces a dar morfologías "blocosas", donde partículas primarias $(<2 \mu \mathrm{m})$ se agrupan en "clusters" dejando entre sí una porosidad destacable (Figura 4). Estos casos pueden evolucionar con los meses de maduración a arquitecturas que hemos denominado "reticuladas" porque semejaban una red, incluso perdiendo en apariencia la identidad física las partículas que aparecían como en continuo. En otras muestras aparecen casos de fábricas en "panal de abejas" ó "castillo de naipes" (Figura 5), en ocasiones con una evolución con el tiempo desde tipos previos. Una fábrica que puede indicar la degradación de la arquitectura es la "laminar masiva" (Figura 6) con pérdida drástica de porosidad.

Figura 4 - Imagen SEM de la muestra A1

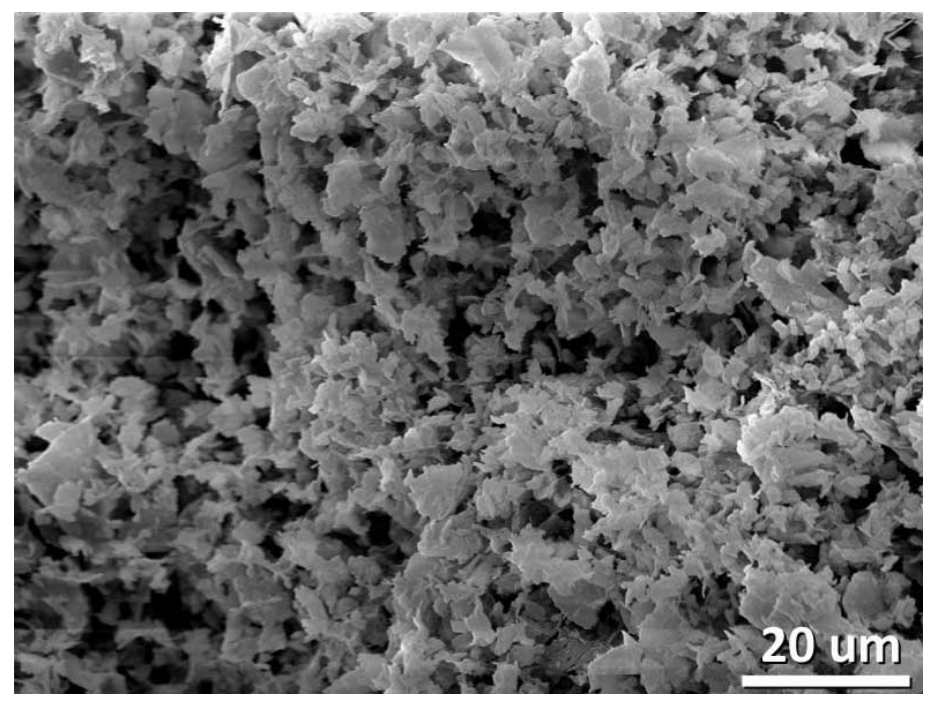

El origen de todos estos tipos es siempre controvertido por su dependencia de múltiples variables, ya que además de las citadas, naturaleza mineralógica de la fase sólida y tiempo de maduración, parece influir, entre otros, el contenido de solutos del agua y su naturaleza. 
Figura 5 - Imagen SEM de la muestra E3

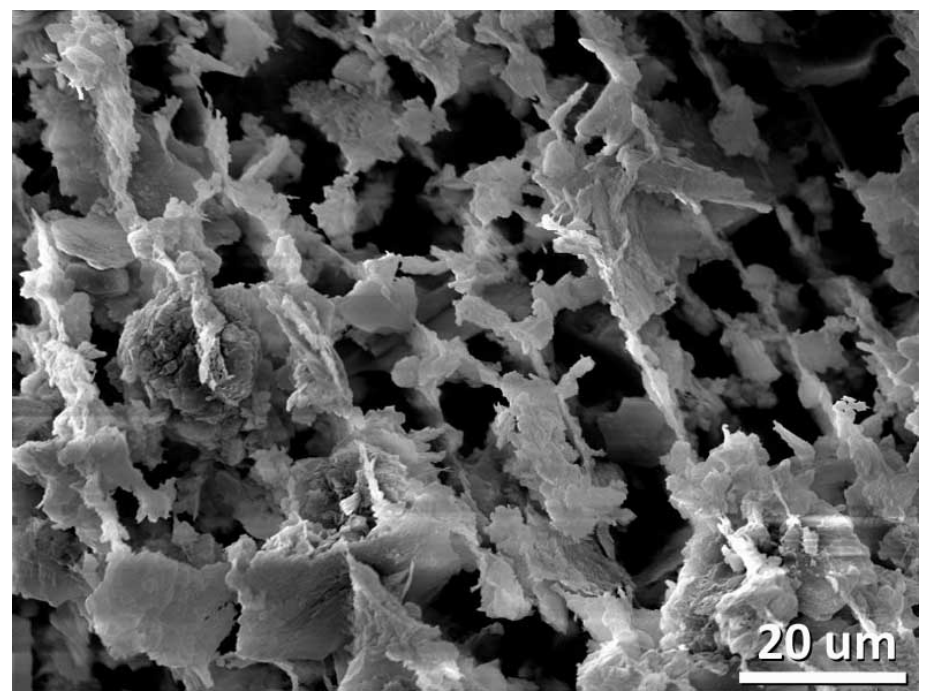

Figura 6 - Imagen SEM de la muestra S6

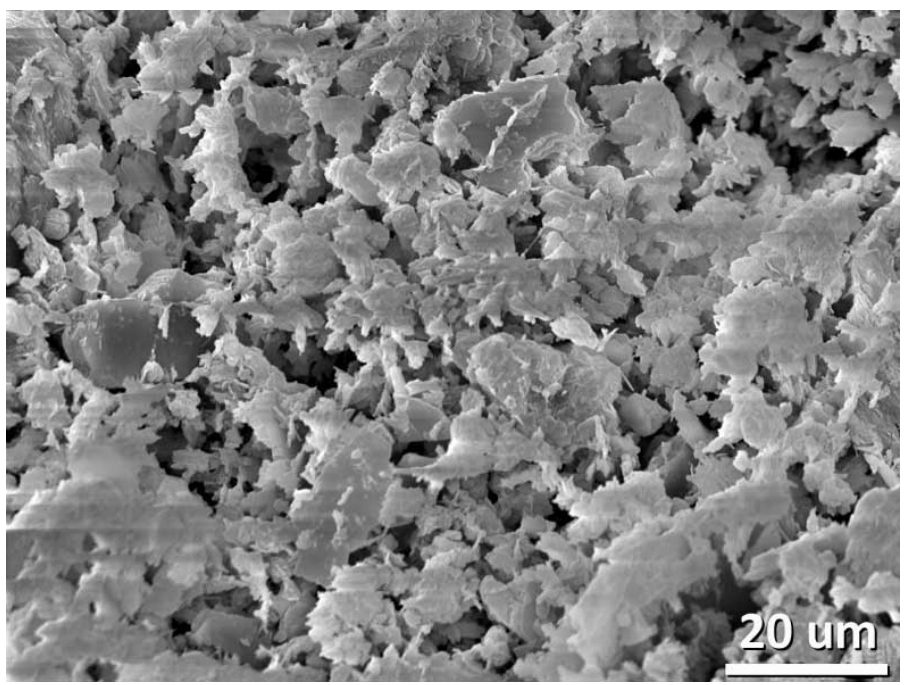

Las porosidades medidas con IA son concordantes con el tipo de fábrica. Así las mayores se registran en las castillo de naipes/ panal de abejas, duplicando y triplicando a la de las laminares masivas. En los casos más favorables, la porosidad supera el $30 \%$ de la masa. 
Considerando que las fábricas se han visto evolucionar en su tipo morfogenético con el tiempo de maduración y degradarse disminuyendo en su porosidad (estimada cuantitativamente con análisis de imagen), hemos marcado para cada tipo de agua mineromedicinal y mineral de las empleadas el tiempo óptimo de maduración. En una mayor frecuencia de casos son los tres meses.

\section{CONCLUSIONES}

El estudio de la ultramicrofábrica mediante SEM, EDX, IA ofrece buenas perspectivas para comprender el proceso de maduración de los peloides.

- La fábrica tiene una evolución detectable con el tiempo, si bien el nivel organizativo de las partículas alcanzado a las 48 horas es importante, aportando un nuevo dato a favor de los peloides extemporáneos.

- En nuestros peloides se han descrito como más importantes tipos morfogenéticos de fábricas laminares dispersas, reticuladas, castillo de naipes/ panal de abejas y tendentes a laminares masivas.

- Los valores de porosidad estimados concuerdan con el tipo de fábrica y en los casos mejor organizados pueden superar el $30 \%$.

- El óptimo de maduración más frecuente, de acuerdo al tipo de fábrica, podemos marcarlo en los tres meses.

\section{BIBLIOGRAFIA}

1. Kubiena WL. Micropedology. Ames, Iowa: Collegiate Press, 1938.

2. Delgado R. Proyecto de investigación "Estudio de suelos rojos". Report interno. 1992.

3. Bullock P, Fedoroff N, Jongerius A, Stoops G, Tursina T. Handbook for soil thin section description. Waine Research Publications, 1985.

4. Bates RL, Jackson JA. Glossary of Geology. Amer. Geol. Institute, Falls Chusch, Va. 1980, 751 pp.

5. Yong RN, Warkentin BP. Soil Properties and Behaviour. Developments, in Geotechnical Engineering, 5, Elsevier Scientific Publishing Company, Amsterdam, Oxford, New York, 1975, 449 pp.

6. Martín-García JM, Aranda V, Gámiz E, Bech J, Delgado R. Are Mediterranean mountains Entisols weakly developed? The case of Orthents from Sierra Nevada (Southern Spain). Geoderma 2004; 118: 115-131.

7. Calero J, Delgado R, Delgado G, Martín-García JM. SEM image análisis in the study of a soil chronosequence on fluvial terraces of the middle Gua- 
dalquivir (Southern Spain). European Journal of Soil Science 2009; 60: 465480.

8. Veniale F, Barberis E, Carcangiu G, Morandi N, Setti M, Tamanini M, Tessier D. Formulation of muds for pelotherapy: effects of "maturation" by different mineral waters. Appl. Clay Sci. 2004; 25: 135-148.

9. Galzigna L, Moretto C, Lalli A. Physical and biochemical change of thermal mud alter maturation. Biomed \& Pharmacother 1996; 50: 306-308.

10. Galzigna L, Ceschi-Berrini C, Moschin E, Tolomeo C. Thermal mud-pack as anti-inflamatory treatment. Biomed \& Pharmacother. 1998; 52: 408-409.

11. Gámiz E, Martín-García JM, Fernández-González MV, Delgado G, Delgado $\mathrm{R}$. Influence of water type and maturation time on the properties of kaolinitesaponite peloids. Appl. Clay Sci. 2009; 46: 117-123.

12. Fernández-González MV, Gámiz E, Martín-García JM, Márquez R, Delgado G, Delgado R. Comportamiento térmico de peloides preparados con aguas mineromedicinales del Balneario de Lanjarón. Efecto del tiempo de maduración. Anal Hidrol Med, 2011, vol. 4, 91-103.

13. National Institutes of Health. Image J. Image Processing and Análisis in Java. 2008. Disponible en: http://rsb.info.nih.gov/ij/.

14. Gámiz E, Linares J, Delgado R. Assessment of two Spanish bentonites for pharmaceutical uses. Appl. Clay Sci. 1992; 6: 359-368.

15. Armijo F, Vela L, Aguilera L, Maraver F. Características físicas del peloide elaborado con el agua mineromedicinal Lanjarón- Salado (Lanjarón- Granada). Libro de resúmenes del II Congreso Iberoamericano de Peloides (Lanjarón). Madrid: Segarsa, 2010, 73-74.

16. Teixeira, F. La utilización de las Lamas en Portugal: passado y presente. Libro de resúmenes del II Congreso Iberoamericano de Peloides (Lanjarón) Madrid: Segarsa, 2010, 14-15. 